\title{
Docentes emocionalmente inteligentes. Importancia de la Inteligencia Emocional para la aplicación de la Educación Emocional en la práctica pedagógica de aula
}

\author{
Emotionally intelligent teachers: importance of Emotional Intelligence for application of \\ Emotional Education in pedagogical work
}

\author{
Claudia Costa-Rodriguez $z^{a}$ Ximena Palma-Leal ${ }^{b}$, Carla Salgado Farías ${ }^{a}$ \\ ${ }^{a}$ Universidad Mayor, Chile. \\ claudia.costa@mayor.cl, carla.salgado@mayor.cl \\ ${ }^{b}$ Grupo IRyS, Escuela de Educación Física, Pontificia Universidad Católica de Valparaíso, Chile. \\ ximena.palmaleal@gmail.com
}

\begin{abstract}
RESUMEN
La presente revisión aborda como temática principal la importancia de la Inteligencia Emocional como requisito fundamental de las y los docentes en su práctica pedagógica, al mismo tiempo que se destaca la Educación Emocional en las y los estudiantes como herramienta fundamental para la mejora de los procesos de enseñanzaaprendizaje y para el desarrollo integral de las personas. Para cumplir este objetivo se comienza con un análisis acabado de las emociones, su procesamiento a nivel cerebral y su incidencia en el proceso de aprendizaje. Posteriormente se realiza una descripción detallada de la relación entre Educación Emocional e Inteligencia Emocional y la formación inicial del profesorado en esta área, finalizando con la revisión de tres programas de formación en Educación Emocional para estudiantes de pedagogía, evidenciando la necesidad de incluir contenidos al respecto tanto en la formación docente como de forma transversal en la educación a nivel escolar y universitaria.
\end{abstract}

Palabras claves: emociones, aprendizaje, docente, sistema escolar, formación integral.

\section{ABSTRACT}

This review analyses, as its main issue, the importance of Emotional Intelligence as essential requirement in pedagogical work of teachers. At the same time its emphasizes Emotional Education of students like a fundamental tool to improve teaching-learning process and for integral development of people. In order to achieve this objective it was made a depth analysis of emotions, their brain processing and their effect in learning process. Subsequently, a detailed description is given of the relationship between Emotional Education and Emotional Intelligence and teacher's initial training in this area. Ending with a review of three training programs of Emotional Education for Pedagogy Students, this evidences the need to include contents for this topic in both, teacher training and transversally in school and university.

Key words: emotions, learning, teacher, school system, comprehensive training. 
Estudios Pedagógicos XLVII Nº 1: 219-233, 2021

DOCENTES EMOCIONALMENTE INTELIGENTES. IMPORTANCIA DE LA INTELIGENCIA EMOCIONAL PARA LA

APLICACIÓN DE LA EDUCACIÓN EMOCIONAL EN LA PRÁCTICA PEDAGÓGICA DE AULA

\section{INTRODUCCIÓN AL TEMA DE ESTUDIO}

Un tema recurrente en Chile hoy en día es la percepción que la educación o el sistema educativo se encuentran en crisis. En este contexto, Claudio Naranjo señala que esta crisis se evidencia tanto en la infelicidad de los y las docentes, como de los estudiantes; "en los docentes tal infelicidad se manifiesta en desmotivación, depresión y enfermedades físicas, en tanto que en los estudiantes se manifiesta en desinterés, rebeldía, trastornos de la atención y del aprendizaje, y violencia" (Naranjo, 2014, p. 14).

Hoy en día, se puede ver cómo en las escuelas se encuentran niños y niñas con diversos diagnósticos que etiquetan dificultades en su comportamiento, el cual no se adecúa o no se logra enmarcar en las normas socialmente aceptadas. En este sentido es posible apreciar que los padres muchas veces intentan moldear a sus hijos de acuerdo a sus expectativas personales, dejando de lado el apoyo y contención emocional que las y los niños requieren en determinadas situaciones (Casassus, 2007). En el contexto escolar, "la escuela es el lugar donde se aprende a vivir y a convivir, (...) el niño aprende a relacionarse con pares y con adultos en una interacción cotidiana e intensa" (Céspedes, 2018, pp. 174-175). De esta manera, entre carencias y moldeamientos, las niñas y niños aprenden a reprimir, controlar y manejar sus emociones de forma inadecuada. Este hecho es particularmente importante en la infancia, ya que se ha visto que la represión de emociones, por tiempo prolongado, puede traer consecuencias negativas para la salud física y emocional.

En el ámbito netamente educativo, es relevante señalar que las emociones son fundamentales en el proceso de enseñanza-aprendizaje por dos motivos:

(...) en primer lugar [porque] el proceso educativo implica la interacción entre personas y, en segundo lugar, porque la identidad personal y profesional de los docentes en muchas ocasiones son inseparables y en el aula se convierten en factores de influencia en la autoestima y en el bienestar personal y social. (Nías 1996, como se citó en Cejudo y López-Delgado, 2017, p. 30).

En este sentido, es importante destacar la importancia que adquiere el rol docente en su práctica pedagógica, ya que su desempeño exige un alto nivel de sensibilidad a las emociones propias y de sus estudiantes, facilitando así una óptima calidad de las relaciones interpersonales que se dan al interior de la escuela.

La práctica profesional se encuentra constantemente controlada por conductas emocionales, de modo que estamos ante una práctica cognitiva y emocional, y "en tanto que seres humanos, los profesores están siempre bajo la influencia de las emociones" (Casassus, 2007, p. 241). Es entonces donde se visualiza la necesidad de que las y los docentes desarrollen necesariamente su Inteligencia Emocional para mejorar los procesos de enseñanza-aprendizaje en sus aulas.

En este ámbito, son varios los investigadores que han señalado la relevancia de que el profesorado comience este camino desde la formación inicial, como parte de las competencias básicas necesarias para el buen desempeño de cualquier docente. Bisquerra (2005) por ejemplo, destaca el hecho de que un profesor puede ser más consciente de los vínculos socio-afectivos que establece con sus estudiantes en la medida que es más consciente de su propia emocionalidad y de la de sus estudiantes. 
Situando el foco en la formación pedagógica de pregrado en Chile, se puede observar que las mallas curriculares están cada vez más orientadas a una concepción tecnicista de la pedagogía, donde los docentes en formación pueden llegar a ser buenos expertos en los contenidos procedimentales de su especialidad; sin embargo, el entramado de la formación inicial, en la mayoría de los casos, no considera los aspectos sociales, afectivos y sobre todo emocionales del proceso de aprendizaje.

Debido a lo anteriormente planteado, en el curso del presente artículo se expondrán los resultados más importantes obtenidos de esta investigación con el fin de entregar evidencia contundente que dé sustento a la importancia del desarrollo de la Inteligencia Emocional en los docentes, para que luego, estos puedan educar emocionalmente a sus estudiantes y mejorar así los procesos de aprendizaje en el aula y en la vida en general.

\section{METODOLOGÍA DE INVESTIGACIÓN}

El presente artículo se realizó a partir de una revisión de diversa bibliografía con enfoque descriptivo y procesamiento documental acerca del objeto de estudio (Hernández-Sampieri, Fernández y Baptista, 2014). Se empleó la consulta, revisión y análisis de textos de autores reconocidos tanto a nivel nacional como internacionalmente con más de 10 años de trayectoria en la temática de la Educación Emocional y/o Inteligencia Emocional. También se seleccionaron artículos de revistas indexadas de los últimos veinte años referentes a la temática de investigación.

Posterior a la fase de revisión, se comenzó la construcción del estado del arte mediante el método de mapeo (Hernández-Sampieri et al., 2014), para finalmente dar paso a la construcción de los resultados y elaboración de las conclusiones, realizando una exhaustiva lectura analítica de toda la información bibliográfica recopilada en el estado del arte.

\section{RESULTADOS}

\subsection{QUÉ SON LAS EMOCIONES Y CUÁL ES SU FUNCIÓN}

Durante la revisión de la bibliografía fue posible constatar la existencia de diversos enfoques desde donde es posible analizar y definir la emoción; sin embargo, aún no existe un consenso generalizado en su definición. Lo que sí se pudo determinar como elemento común en todas las definiciones, es el hecho de que los autores señalan que la emoción (o las emociones) está siempre a la base de una acción o movilización del individuo.

Desde los más antiguos pensadores, la emoción se presenta como un concepto complejo de definir, Aristóteles lo describió como una "condición que transforma a la persona de tal manera que puede verse afectado el juicio" (Bisquerra, 2010, p. 29). Posteriormente a mediados del siglo XIX, Charles Darwin publicó en 1872, La Expresión de las Emociones en Hombres y Animales, en el cual declaró que "las emociones son reacciones adaptativas y necesarias para la supervivencia. (...) Tienden a ser reacciones apropiadas a la emergencia ante ciertos acontecimientos del entorno" (Bisquerra, 2010, p. 35). Luego ya a finales del siglo XX, Daniel Goleman, psicólogo y periodista estadounidense, fue pionero en realizar publicaciones acerca de la incipiente Inteligencia Emocional. Él se aventuró en decir que 
la emoción se refiere a "un sentimiento y a los pensamientos, los estados biológicos, los estados psicológicos y el tipo de tendencias a la acción que los caracterizan" (Goleman, 1996, p. 182). A continuación, en 2001, el destacado biólogo y filósofo chileno Humberto Maturana describió las emociones desde una perspectiva biológica, definiéndolas como "disposiciones corporales que determinan o especifican dominios de acciones" (Maturana, 2001, p. 8); también señala que no es posible ninguna acción humana sin una emoción que la funde como tal y la haga posible como acto o acción. Y finalmente en 2007, el filósofo y sociólogo chileno Juan Casassus (2007), las definió como una energía vital que va más allá de una experiencia psicológica o biológica, “(...) es un tipo de energía que une los acontecimientos externos con los internos (...), de internalización y externalización unidos por una energía que es una disposición a actuar" (p. 99). Finalmente, el psicólogo argentino Lucas Malaisi (2016) las define como un "estado psico-biológico que brinda información y energía existencial, afectando profundamente el desempeño personal" (p. 59), además agrega que son el motor del hombre que moviliza los medios para la satisfacción de las necesidades.

En cuanto a la función de las emociones, fue posible referir la función adaptativa como una de las principales y más importantes finalidades. En este ámbito, es importante destacar que, desde las épocas primitivas, las emociones han sido las responsables de preservar la especie humana, y esto se fundamenta en que emociones como el miedo, por ejemplo, fueron la base para que los seres humanos fueran capaces de generar respuestas como la huida o la defensa frente a peligros inminentes como animales o desastres naturales. Desde esta misma perspectiva, Casassus (2007) señala que "las emociones están a la base de la acción (...), puesto que están basadas en la relación entre eventos y nuestras intenciones e intereses, nos permiten adaptarnos a las situaciones en las que nos encontramos" (p. 101). $\mathrm{El}$ autor afirma que las emociones y sentimientos son fundamentales para la supervivencia del hombre ya que guían los actos humanos de acuerdo a los principios básicos para la vida: la auto-conservación y la preservación de la especie.

En segunda instancia, se pudo evidenciar que otras funciones también muy importantes se relacionan con múltiples y variados aspectos de la vida cotidiana de los seres humanos: informan al individuo de acontecimientos internos y externos, comunican socialmente estados de ánimo, afectan en variados procesos mentales y en la toma de decisiones (Bisquerra, 2010).

\subsection{EL CEREBRO EMOCIONAL}

Para comprender el funcionamiento emocional es necesario recordar que el cerebro humano ha ido creciendo y modificándose a lo largo de millones de años de evolución. La región más primitiva del cerebro, en la cual se encuentran estructuras como el tallo encefálico, cumple funciones vitales básicas para la supervivencia de los individuos. En este cerebro primitivo, se puede encontrar también la aparición del sistema límbico rodeando el tronco (o tallo) encefálico, "este nuevo territorio neural agregó las emociones propiamente dichas al repertorio de respuestas del cerebro" (Goleman, 1996, p. 13). Durante este proceso evolutivo de las estructuras cerebrales, "emergieron los centros emocionales que, millones de años más tarde, dieron lugar al cerebro pensante o neocortex" (Goleman, 1996, p. 13).

Desde la perspectiva anatómica, el sistema límbico es la zona subcortical del cerebro conformada por un conjunto de estructuras que rodean al tálamo entre las cuales se 
encuentran la amígdala, el hipotálamo, el hipocampo, los cuerpos mamilares, el fórnix, la corteza del cíngulo, el septum y el bulbo olfatorio.

En la Figura $n^{\circ} 1$ es posible ver con mayor claridad las estructuras cerebrales recién mencionadas.

Dentro de las estructuras del cerebro emocional, la amígdala cerebral tiene una función esencial en la activación de la respuesta emocional, funcionando como un computador que evalúa y hace juicios del contenido emocional de los estímulos que recibe; "es la amígdala la que transmite una señal no verbal, sino eléctrica a otras partes del cerebro que alteran la atención e inician respuestas fisiológicas al evento que ha gatillado la reacción" (Casassus, 2007, p. 106). La psiquiatra chilena Amanda Céspedes también explica que es la responsable de otorgar "valencia a los cambios internos generados por estímulos externos" (Céspedes, 2008, p. 205), permitiendo adjetivar y clasificar las emociones en positivas y negativas.

Figura 1. Estructuras cerebrales implicadas en la respuesta emocional.

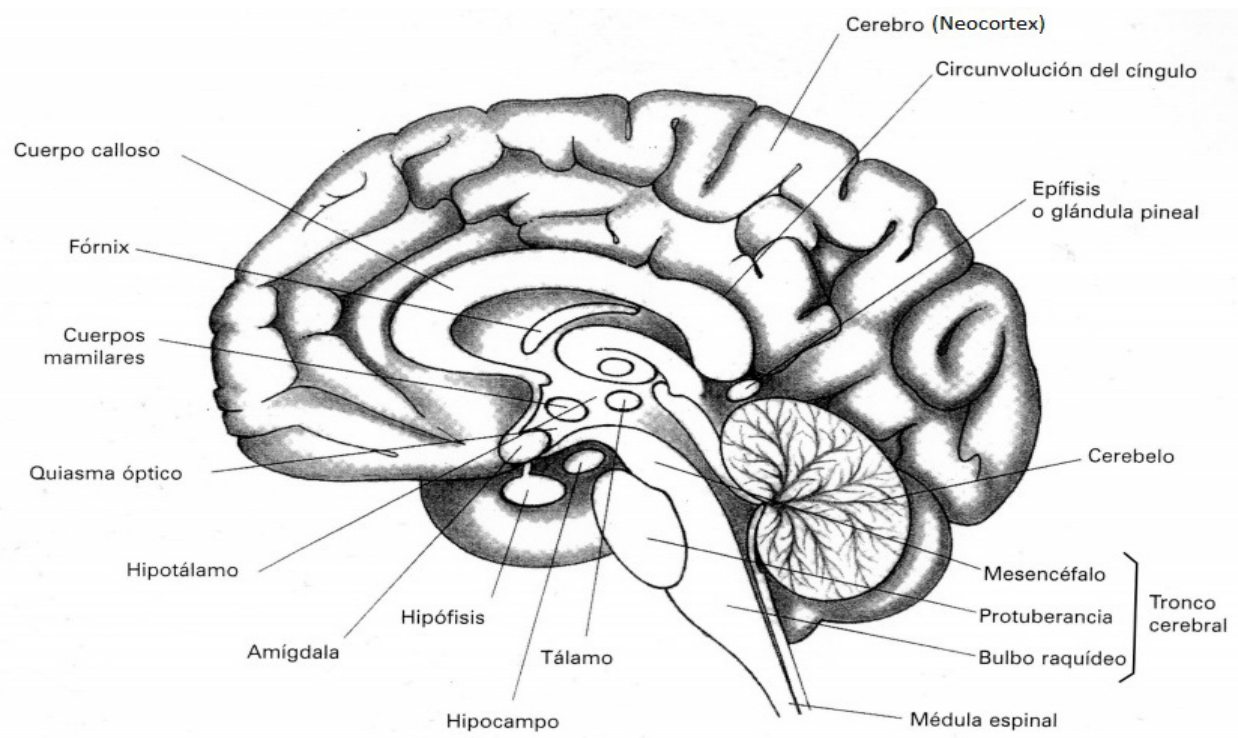

Recuperado de "Psicopedagogía de las emociones". Bisquerra, R., 2010, p. 99.

Madrid, España: Editorial Síntesis.

\subsection{EMOCIONES EN EL APRENDIZAJE}

En el ámbito de las emociones relacionadas con el aprendizaje, fue posible establecer que las emociones son la base del aprendizaje, e incluso afirmar que "no hay aprendizaje fuera del espacio emocional" (Casassus, 2007, p. 239). En este sentido es importante precisar que las emociones pueden facilitar u obstaculizar el aprendizaje, lo cual depende del ambiente o contexto emocional que sea capaz de crear el docente, incentivando la 
participación activa de los estudiantes y generando una disposición emocional positiva hacia el aprendizaje.

Al proceso de aprendizaje basado en las emociones Malaisi (2016) le llama anclaje, y este se produce cuando el o la docente es capaz de generar en el aula situaciones que produzcan emociones intensas. El autor hace referencia al Cono del Aprendizaje de Edgar Dele (p. 115), el cual ilustra la efectividad de diferentes formas de aprendizaje, donde se destaca que luego de dos semanas los estudiantes usualmente recuerdan entre el $70 \%$ y 90\% de lo que aprendieron mediante la vivencia de experiencias reales, de simulación, dando una charla o participando en una discusión, es decir, mediante la participación activa en sus procesos de aprendizaje. Este porcentaje baja drásticamente cuando la participación de las y los estudiantes es pasiva, llegando a recordar el 30\% de lo aprendido en actividades como ver imágenes o solamente escuchando a los docentes.

En este contexto entonces, adquiere gran importancia la labor docente en cuanto a generar un ambiente de aprendizaje que, por una parte, motive la participación activa de los y las estudiantes en las tareas propuestas, y que paralelamente genere una disposición emocional positiva, ya que estas son determinantes para facilitar u obstaculizar los aprendizajes (García, 2012).

\subsection{EDUCACIÓN EMOCIONAL E INTELIGENCIA EMOCIONAL}

La realidad actual de las escuelas en Chile es que las y los estudiantes pasan alrededor de ocho horas diarias en las dependencias educacionales. Es allí donde reciben permanentes influencias y enseñanzas de parte de sus pares, profesores, profesoras y otros adultos. Es por esto que se torna fundamental comprender la tarea transversal que realizan las y los docentes en la vida de los niños y niñas que educan a diario, donde el desarrollo de habilidades emocionales y socio-afectivas sea probablemente lo que determine la capacidad que tengan los estudiantes de poner en práctica con éxito los conocimientos académicos y habilidades cognitivas alcanzadas en la escuela. Debido a lo anterior, es que se hace necesario comenzar por describir y comprender el concepto de Educación Emocional (en adelante EE) e Inteligencia Emocional (en adelante IE), y la relación entre ambos.

A partir de los resultados obtenidos en esta investigación, fue posible establecer que la finalidad de la EE es educar para la vida, lo cual implica que a partir del desarrollo de habilidades emocionales y sociales el individuo pueda aumentar su bienestar personal y social, considerando que este proceso se realiza a lo largo de todo el ciclo vital. Los autores coinciden en considerar que la EE debe abordarse como una forma de prevención primaria inespecífica ya que gracias a ella es posible la adquisición de competencias que se pueden aplicar a una diversidad de situaciones durante toda la vida, minimizando la vulnerabilidad del individuo frente a dichas circunstancias, como por ejemplo consumo de drogas, prevención del estrés, ansiedad, depresión, impulsividad, frustración, etcétera (Bisquerra, 2010; Malaisi, 2016). Es importante señalar que la EE se debe instalar primeramente en la formación inicial de las y los docentes para que, posteriormente, estos puedan acompañar a sus estudiantes en este proceso durante todo el ciclo escolar, donde dependiendo del nivel educativo en que se desempeñen, los contenidos de la EE irán variando. Sin embargo, a modo general se pueden destacar los siguientes objetivos de este proceso: 
- Adquirir un mejor conocimiento de las propias emociones.

- Identificar las emociones de los demás.

- Denominar a las emociones correctamente.

- Desarrollar la habilidad para regular las propias emociones.

- Subir el umbral de tolerancia a la frustración.

- Prevenir los efectos nocivos de las emociones negativas.

- Desarrollar la habilidad para generar emociones positivas.

- Desarrollar la habilidad de automotivarse.

- Adoptar una actitud positiva ante la vida.

- Aprender a fluir (Bisquerra et al., 2012, pp. 27-28).

En cuanto a la IE, se definió como la habilidad para percibir, valorar y expresar adecuadamente una emoción. Su desarrollo implica el aprendizaje de ciertas competencias relacionadas con la habilidad para comprender el origen de la emoción y la regulación de la misma con el fin de generar un crecimiento intelectual y emocional (Mayer y Salovey, 1997, como se citó en Bisquerra, 2010). En este contexto cabe señalar que con frecuencia se considera como inteligente a aquella persona que domina varias áreas de conocimiento, que posee títulos académicos o que habla distintos idiomas. Sin embargo, la palabra correcta para este tipo de personas sería intelectual: "dedicado preferentemente al cultivo de las ciencias y las letras" (RAE, 2019). Una persona inteligente es aquella que tiene capacidad de entender o comprender, pero sobre todo es alguien con capacidad de resolver problemas, que sabe escoger asertivamente entre varias opciones (Malaisi, 2016). En este sentido, el tener a disposición un buen registro de las emociones experimentadas, indudablemente posiciona de mejor forma al individuo para poder elegir entre varias opciones con mayor seguridad.

En el contexto de la labor docente, la práctica profesional está marcada por patrones de conducta emocionales que la mayoría de las veces ocurren de forma inconsciente, por lo tanto, el nivel de IE que el profesor o profesora pueda desarrollar es de vital importancia para generar un buen clima de aula. En este sentido Casassus (2007) señala que "un profesor es más consciente de sus vínculos, y de los patrones de relación que establece, siendo más consciente de su propia emocionalidad y de la de sus alumnos" (p. 244). Esta conciencia emocional le permitirá innovar en viejos y deficientes patrones al mismo tiempo que valorar aquellos aspectos que sí fortalecen los vínculos con sus estudiantes.

Gracias a lo anteriormente planteado, se pudo evidenciar que los objetivos de la EE se relacionan estrechamente con las competencias emocionales necesarias para el logro de la IE, por lo tanto, se entiende que la IE aparece como el fin último que persigue la EE.

\subsection{DOCENTES EMOCIONALMENTE INTELIGENTES}

Como se ha evidenciado anteriormente, la EE se fundamenta en objetivos y competencias emocionales que apuntan hacia una educación que traspasa los ámbitos puramente académicos; más lejos, se enmarca en una perspectiva de desarrollo humano que busca la construcción de las bases de una educación que prepare para la vida. Es en esta instancia donde aparecen las y los docentes como los profesionales más idóneos para implementar la EE en las escuelas.

Si se considera que el aprendizaje escolar es una actividad social constructiva en la cual participan las y los educandos guiados por el o la docente, es clave considerar que 
Estudios Pedagógicos XLVII Nº 1: 219-233, 2021

DOCENTES EMOCIONALMENTE INTELIGENTES. IMPORTANCIA DE LA INTELIGENCIA EMOCIONAL PARA LA

APLICACIÓN DE LA EDUCACIÓN EMOCIONAL EN LA PRÁCTICA PEDAGÓGICA DE AULA

este no enseña ni se desenvuelve de manera abstracta dentro del aula, dejando de lado sus propias emociones y sentimientos, "sino que, ya sea de manera explícita o implícita, transmite los mismos -emociones y sentimientos- en cada acto pedagógico que desarrolla" (García, 2012, p. 14). En este sentido es claro que el papel del docente es fundamental en la promoción de habilidades tanto cognitivas como emocionales que permitan un aprendizaje autónomo en sus estudiantes, el cual pueda ser utilizado en la resolución de problemáticas y conflictos en cualquier ámbito de la vida, no solo en el contexto escolar.

La psiquiatra chilena Amanda Céspedes (2008) señala que para que el profesor pueda llevar a cabo una EE efectiva debe poseer las siguientes características:

- Conocimiento intuitivo o informado acerca de la edad infantil y adolescente.

- Conocer la importancia de los ambientes emocionalmente seguros en el desarrollo de la afectividad infantil.

- Razonable equilibrio psicológico y ausencia de psicopatología.

- Técnicas efectivas de afrontamiento de conflictos.

- Comunicación afectiva y efectiva.

- Permanente y sincero trabajo de autoconocimiento.

- Reflexión crítica constante acerca de los sistemas de creencias y de su misión como educador (pp. 142-143).

Dado lo anterior, es posible visualizar que la capacidad para identificar, comprender y regular las emociones son habilidades fundamentales entre el profesorado, ya que además de influir en los procesos de aprendizaje, "afectan la salud física, la calidad de las relaciones interpersonales y el rendimiento académico y laboral" (Cabello, Ruiz-Aranda y Fernández-Berrocal, 2010, p. 4) del docente en ejercicio. Lo anterior indica que el ambiente de aprendizaje que el profesor sea capaz de generar depende en gran parte de su IE, es decir, el hecho de que los profesores aprendan a generar, regular y mantener en sí mismos estados emocionales positivos, reduciendo el impacto de los negativos, puede verse reflejado en un mayor bienestar docente y por ende, en estudiantes emocionalmente más preparados y dispuestos positivamente al aprendizaje y a enfrentar de mejor manera los eventos conflictivos o desafiantes que surgen con frecuencia en el contexto educativo.

\subsection{FORMACIÓN INICIAL DEL PROFESORADO EN EDUCACIÓN EMOCIONAL}

La puesta en práctica de la EE requiere de una formación previa del profesorado, lo cual evidenció uno de los resultados más importantes de esta investigación, la necesidad de que las y los profesores adquieran una formación previa en EE, idealmente en la etapa de pregrado, con el fin de que puedan alcanzar un óptimo desarrollo de su IE antes de comenzar el acompañamiento a sus estudiantes en este mismo proceso. Sin embargo, cabe señalar que, aunque en el transcurso de la recopilación bibliográfica se encontraron variados programas de EE que apuntan al desarrollo de la misma en los estudiantes, no se encuentra tal variedad y cantidad de programas que orienten la formación del profesorado en el desarrollo de sus propias competencias emocionales, proceso indispensable para que luego las y los docentes puedan guiar a sus estudiantes en la educación de sus emociones.

Entendiendo la importancia de apuntar primeramente a la formación del profesorado en EE, a continuación, se presentan tres programas o modelos de formación inicial, los 
cuales fueron seleccionados bajo el criterio de escoger aquellas propuestas que se orienten específicamente al desarrollo de competencias emocionales en docentes.

\subsubsection{Propuesta de Rafael Bisquerra (2005)}

Este programa parte de la base de que en la formación inicial (universitaria), las y los estudiantes de pedagogía deberían recibir una formación que los dotara de un bagaje sólido en materia de emociones y sobre todo en competencias emocionales. Lo anterior se fundamenta en que estos conocimientos permitirán al maestro afrontar mejor la tarea educativa en toda su complejidad, comenzando por su propio desarrollo personal y profesional y desde allí por extensión se potenciará el desarrollo del alumnado. Es así que este programa propone una asignatura sobre EE que se enmarca en la formación inicial del profesorado para educación infantil, primaria y secundaria (Bisquerra, 2005).

A continuación, se presenta la planificación curricular que se propone para la asignatura. Cabe señalar que el siguiente cuadro ha sido diseñado por las autoras de esta investigación con el fin de optimizar la información y lograr una mayor comprensión de la misma.

Cuadro 1. Planificación curricular programa EE para formación inicial docente

\begin{tabular}{|c|c|}
\hline & opuesta asignatura Educación Emocional para profesores en formación inicial \\
\hline & : Al final de la asignatura el alumnado habrá adquirido competencias para: \\
\hline 1 & Comprender la relevancia de las emociones en la educación y en la vida diaria. \\
\hline 2 & $\begin{array}{l}\text { Tomar conciencia de las propias emociones y de las de los demás, en particular del alumnado } \\
\text { en las interacciones sociales. }\end{array}$ \\
\hline 3 & $\begin{array}{l}\text { Regular las propias emociones ejerciendo autocontrol emocional en las situaciones } \\
\text { conflictivas de la vida ordinaria, en particular en la dinámica de la clase. }\end{array}$ \\
\hline 4 & Establecer mejores relaciones interpersonales en el trabajo, familia y relaciones sociales. \\
\hline 5 & $\begin{array}{l}\text { Enfrentarse al alumnado de forma más eficiente y satisfactoria, tomando en consideración la } \\
\text { dimensión emocional. }\end{array}$ \\
\hline 6 & $\begin{array}{l}\text { Realizar un análisis del contexto para identificar necesidades emocionales que deberán ser } \\
\text { atendidas a través de un programa de Educación Emocional. }\end{array}$ \\
\hline 7 & $\begin{array}{l}\text { Formular objetivos de un programa de Educación Emocional cuyo logro suponga la } \\
\text { satisfacción de las necesidades prioritarias. }\end{array}$ \\
\hline 8 & $\begin{array}{l}\text { Determinar posibles indicadores que permitan diseñar estrategias de intervención, así como } \\
\text { los aspectos en los que debe incidir la evaluación del programa. }\end{array}$ \\
\hline 9 & Diseñar un programa de desarrollo de las competencias emocionales en el alumnado. \\
\hline 10 & Diseñar estrategias y actividades para aplicarlas en la puesta en práctica del programa. \\
\hline 11 & $\begin{array}{l}\text { Aplicar el programa a un grupo clase, preferentemente en el marco del Plan de Acción } \\
\text { Tutorial. }\end{array}$ \\
\hline 12 & orados previamente. \\
\hline
\end{tabular}


Estudios Pedagógicos XLVII N ${ }^{\circ}$ : 219-233, 2021

DOCENTES EMOCIONALMENTE INTELIGENTES. IMPORTANCIA DE LA INTELIGENCIA EMOCIONAL PARA LA APLICACIÓN DE LA EDUCACIÓN EMOCIONAL EN LA PRÁCTICA PEDAGÓGICA DE AULA

\begin{tabular}{|c|c|c|c|c|c|c|}
\hline \multicolumn{7}{|l|}{ Contenidos: } \\
\hline $\begin{array}{c}\text { Marco } \\
\text { conceptual de } \\
\text { las emociones }\end{array}$ & $\begin{array}{l}\text { Tipología } \\
\text { de las } \\
\text { emociones }\end{array}$ & $\begin{array}{c}\text { Teorías } \\
\text { de las } \\
\text { emociones }\end{array}$ & $\begin{array}{c}\text { Cerebro } \\
\text { emocional }\end{array}$ & $\begin{array}{c}\text { Emociones } \\
\text { y salud }\end{array}$ & $\begin{array}{c}\text { Aportaciones } \\
\text { de la terapia } \\
\text { emocional }\end{array}$ & $\begin{array}{c}\text { Inteligencias } \\
\text { múltiples }\end{array}$ \\
\hline $\begin{array}{l}\text { Inteligencia } \\
\text { Emocional }\end{array}$ & $\begin{array}{l}\text { Características } \\
\text { de la } \\
\text { Educación } \\
\text { Emocional }\end{array}$ & $\begin{array}{l}\text { Conciencia } \\
\text { emocional }\end{array}$ & $\begin{array}{l}\text { Regulación } \\
\text { emocional }\end{array}$ & $\begin{array}{l}\text { Emoción y } \\
\text { bienestar } \\
\text { subjetivo }\end{array}$ & Fluir (flow) & $\begin{array}{c}\text { Aplicaciones } \\
\text { de la } \\
\text { Educación } \\
\text { Emocional }\end{array}$ \\
\hline \multicolumn{7}{|l|}{ Metodología } \\
\hline \multicolumn{3}{|c|}{$\begin{array}{ll}\text { - } & \text { Dinámicas de grupo. } \\
\text { - } & \text { Grupos de discusión. } \\
\text { - } & \text { Juegos de rol. }\end{array}$} & \multicolumn{4}{|c|}{$\begin{array}{l}\text { - Trabajo individual continuo de lectura de } \\
\text { documentos, libros, capítulos de libros } \\
\text { seleccionados, artículos, etcétera. } \\
\text { - } \quad \text { Exposiciones magistrales. }\end{array}$} \\
\hline
\end{tabular}

Elaboración propia con datos de "La Educación Emocional en la formación del profesorado". Bisquerra, R., 2005, pp. 101-103. Revista Electrónica Interuniversitaria de Formación del Profesorado.

\subsubsection{Propuesta de Pablo Palomero (2009)}

La propuesta de este psicólogo español parte de un enfoque cualitativo que encuentra su origen en la psicología humanista, poniendo énfasis en el desarrollo de las fortalezas de los individuos, es decir que "pretende facilitar que aquellas personas a las que estamos formando puedan optimizar sus propios recursos para alcanzar sus objetivos y afrontar con éxito las situaciones que se les presentan" (Palomero, 2009, p. 148).

En cuanto a los contenidos del modelo, estos se organizan en teóricos, prácticos y actitudinales, otorgando gran relevancia a estos últimos ya que se considera que tienen gran influencia en la práctica docente y además según estudios del autor, es uno de los aspectos más descuidados en la formación docente.

La propuesta de formación contempla el desarrollo de cinco actitudes que se consideran como indispensables que el profesorado cultive para poder ampliar su competencia emocional y social:

\section{Cuadro 2. Contenidos actitudinales}

\begin{tabular}{|l|l|}
\hline Actitudes & Estrategias de desarrollo \\
\hline $\begin{array}{l}\text { Disposición fenomenológica: capacidad para } \\
\text { percibir el propio mundo cognitivo-emocional. }\end{array}$ & $\begin{array}{l}\text { Mediante dinámicas que permitan que el docente } \\
\text { descubra actitudes y aspectos de su práctica } \\
\text { profesional sobre los cuales no había reparado. }\end{array}$ \\
\hline $\begin{array}{l}\text { Autonomía: Capacidad de dirigir su propio } \\
\text { comportamiento en el aula y centro educativo } \\
\text { (docentes incapaces de dirigir el proceso de } \\
\text { enseñanza-aprendizaje). }\end{array}$ & $\begin{array}{l}\text { Role playing o análisis de casos. En pos de } \\
\text { fomentar la autonomía, no se busca entregar una } \\
\text { solución concreta a la problemática, sino que } \\
\text { clarificar las causas y generar posibles } \\
\text { soluciones. }\end{array}$ \\
\hline
\end{tabular}




\begin{tabular}{|l|l|}
\hline $\begin{array}{l}\text { Responsabilidad: capacidad del docente para } \\
\text { responder por sus propios sentimientos, } \\
\text { pensamientos y comportamientos. }\end{array}$ & $\begin{array}{l}\text { Técnicas de psicoterapia como expresión en } \\
\text { primera persona, clarificación de la vivencia, } \\
\text { reflejo empático, etcétera. }\end{array}$ \\
\hline $\begin{array}{l}\text { Independencia de criterio: capacidad de } \\
\text { construir su identidad profesional a partir de su } \\
\text { personalidad, experiencia y formación. }\end{array}$ & $\begin{array}{l}\text { Reconocer sus puntos fuertes y débiles, explorar } \\
\text { los límites de lo que se es capaz de hacer y } \\
\text { hacerse responsable de sus decisiones. }\end{array}$ \\
\hline $\begin{array}{l}\text { Disposición cooperadora: conciencia de su } \\
\text { pertenencia al grupo, de los valores y objetivos } \\
\text { que comparte con su unidad educativa. Tomar } \\
\text { conciencia de su importancia en el ámbito de la } \\
\text { docencia. }\end{array}$ & $\begin{array}{l}\text { Métodos activos y libertad de expresión, } \\
\text { compartir afectos y opiniones durante los cursos } \\
\text { facilita el sentido de pertenencia. }\end{array}$ \\
\hline
\end{tabular}

Elaboración propia con datos de "Desarrollo de la competencia social y emocional del profesorado: aproximación desde la psicología humanista”, Palomero, P., 2009, pp. 149-152. Revista Electrónica Interuniversitaria de Formación del Profesorado.

Finalmente, el autor recalca que el grupo de participantes y la dinámica generada en él "son la herramienta fundamental para comprender las dimensiones sociales y emocionales de la práctica docente, enfatizando así el carácter relacional de los procesos educativos" (Palomero, 2009, p. 148).

\subsubsection{Propuesta Fundación Liderazgo Chile (2018)}

La Fundación Liderazgo Chile se propone como objetivo principal promover e impulsar la mejora en la calidad de los procesos formativos en el sistema educativo chileno a través de la EE. En este contexto, la institución reconoce la problemática social que significa la falta de EE en Chile y cómo esta falencia afecta a la ciudadanía en todos los ámbitos de desarrollo. Desde esa perspectiva, la fundación formula diversas propuestas entre las cuales se incluye una Ley de Educación Emocional y una propuesta de intervención en establecimientos educacionales.

Es importante señalar que esta propuesta ha sido seleccionada ya que si bien no es un programa orientado exclusivamente a la formación de profesores en EE, sino que es más bien un programa de implementación de EE para centros educativos, se ha escogido por la importante labor que ha realizado la fundación desde hace ya varios años en cuanto a la difusión y promoción de la importancia que tiene la EE en los procesos de aprendizaje y la calidad de vida de la ciudadanía en general, convirtiéndose en una de las agrupaciones con más presencia a nivel nacional e internacional.

La Propuesta de Intervención y Piloto de Educación Emocional en Chile busca integrarse en las escuelas del país de forma permanente generando cambios a largo plazo. Dicho proceso se enmarca dentro de los siguientes lineamientos: 
Estudios Pedagógicos XLVII N ${ }^{\circ}$ : 219-233, 2021

DOCENTES EMOCIONALMENTE INTELIGENTES. IMPORTANCIA DE LA INTELIGENCIA EMOCIONAL PARA LA

APLICACIÓN DE LA EDUCACIÓN EMOCIONAL EN LA PRÁCTICA PEDAGÓGICA DE AULA

Cuadro 3. Objetivos y resultados esperados Propuesta de Intervención

en EE Fundación Liderazgo Chile

\begin{tabular}{|l|l|}
\hline Objetivos & Resultados esperados \\
\hline $\begin{array}{l}\text { Comprender la importancia del desarrollo del } \\
\text { autoconocimiento y sus beneficios en los } \\
\text { procesos formativos de los estudiantes }\end{array}$ & $\begin{array}{l}\text { Establecimientos educativos con reglamentos } \\
\text { que integren un enfoque emocional. }\end{array}$ \\
\hline $\begin{array}{l}\text { Generar instancias de formación docente que } \\
\text { releven a importancia de la EE en las prácticas } \\
\text { pedagógicas. }\end{array}$ & $\begin{array}{l}\text { Disminuir los índices de bullying en las aulas de } \\
\text { las escuelas chilenas. }\end{array}$ \\
\hline $\begin{array}{l}\text { Relevar la implicancia de las interacciones } \\
\text { docente-estudiante, comprendiendo su efecto } \\
\text { en la vida de los educandos. }\end{array}$ & $\begin{array}{l}\text { Adultos comprometidos y participativos en } \\
\text { acciones concretas en el desarrollo de las } \\
\text { relaciones socio-afectivas. }\end{array}$ \\
\hline $\begin{array}{l}\text { Adquirir competencias emocionales y } \\
\text { vincularlas con el proceso enseñanza- } \\
\text { aprendizaje. }\end{array}$ & $\begin{array}{l}\text { Planificaciones estratégicas de } \\
\text { establecimientos que contemplen desarrollo } \\
\text { emocional. }\end{array}$ \\
\hline \begin{tabular}{l} 
Mejorar los procesos de enseñanza-aprendizaje. \\
\hline
\end{tabular}
\end{tabular}

Elaboración propia con datos de "Análisis, fundamento y programa inicial del proyecto de Ley de Educación Emocional en Chile”. Fundación Liderazgo Chile, 2018, p. 59.

El plan de intervención abarca la educación parvularia, enseñanza básica y media y se organiza en cuatro etapas que incluyen a todos los integrantes de la comunidad educativa: familias, directivos, docentes, asistentes de la educación y estudiantes.

Dentro del proceso total que implica un acompañamiento durante cuatro años, se destaca que cada plan de intervención será distinto para cada escuela, ya que durante la primera etapa se realiza una evaluación inicial que busca recoger e identificar las necesidades y problemáticas específicas del establecimiento. Junto con la primera etapa se inicia un periodo transversal a las cuatro etapas que tiene como objetivo sensibilizar a la comunidad educativa acerca de la EE y los beneficios de la misma.

Posteriormente se realiza el diseño y planificación de la intervención mediante jornadas teórico-experienciales tanto para las y los trabajadores del establecimiento como para las familias. La tercera etapa es eminentemente práctica ya que, a través de las diversas asignaturas, las y los profesores comenzarán a implementar en sus estudiantes el desarrollo y aprendizaje de habilidades socio-emocionales. Cabe destacar que en este proceso los docentes recibirán un acompañamiento permanente por parte de especialistas además de asistir a reuniones donde compartirán estrategias con sus pares. En la cuarta y última etapa se realiza una evaluación final acerca de la implementación del programa en la escuela, proyecciones y posibles mejoras. 


\section{DISCUSIÓN Y CONCLUSIONES}

El estudio buscó evidenciar a través de la recopilación de la información bibliográfica presentada, la importancia del desarrollo de la IE en los docentes para la aplicación de la EE en su práctica pedagógica. En este contexto, resulta trascendental establecer que la emoción como base de la acción es una premisa muy importante ya que permite entender que todas las acciones humanas están basadas en respuestas emocionales. De esta manera, lo que marca la diferencia entre la acción que realiza una persona y otra frente al mismo estímulo es el factor cognitivo que ha sido determinado por la forma en que esa persona ha sido educada emocionalmente a lo largo de su vida. En relación a este tema, Casassus (2007) señala que "los eventos exteriores, no determinan, sino que gatillan distintas reacciones en distintas personas. (...) Las emociones son gatilladas por evaluaciones cognitivas de acontecimientos externos; evaluaciones que pueden ser conscientes o inconscientes" (p. 105).

En cuanto a la función de las emociones en el desarrollo del ser humano, es importante señalar que aunque en épocas primitivas tuvieron una función fundamentalmente adaptativa para la supervivencia de la especie, hoy en día se puede ver cómo esa característica ha evolucionado hacia una tarea más de tipo comunicacional, donde el entorno social y educativo adquiere relevancia toda vez que sea necesario que el individuo pueda reconocer, consciente o inconscientemente, los factores externos e internos que lo afectan para posteriormente generar una acción adaptativa a las necesidades propias y del ambiente donde se desenvuelve. En este contexto, se propone como un requisito indispensable para la innovación pedagógica el que todo agente educativo conozca y entienda cómo aprende el cerebro, cómo procesa la información y cómo regula las emociones, sentimientos y estados de ánimo (Campos, 2010). Considerando lo anteriormente planteado, la relación entre emociones y aprendizaje adquiere total validez y relevancia; las emociones son la base para el aprendizaje y muy difícilmente podría asimilarse algún contenido fuera del espacio emocional. En este contexto es importante destacar la importancia de que las y los profesores tengan un acabado conocimiento de su propia emocionalidad y cómo autorregularse, para posteriormente y a partir de sus propias herramientas poder educar y orientar emocionalmente a sus estudiantes.

Los resultados de esta investigación permiten evidenciar la importancia de comprender que, al educar las respuestas emocionales en función del bienestar humano, se puede modificar positivamente el rumbo de las decisiones que toma una persona en su vida. En este sentido, se hace necesario destacar que las primeras dos décadas de vida de la mayoría de las personas se desarrollan principalmente dentro de las escuelas, por lo tanto, parece lo más lógico que, además de las familias, sean los y las docentes los encargados de educar desde la etapa infantil el autoconocimiento y el manejo emocional; sin embargo, esta importante tarea no se debe dejar al criterio o iniciativa de cada profesor o profesora.

Evidentemente, la puesta en práctica de la EE requiere de una formación previa del profesorado; no obstante, durante la búsqueda y selección de las fuentes de información pertinentes para esta investigación fue posible constatar que el desarrollo de competencias emocionales en forma intencionada y sistemática está bastante ausente en las mallas curriculares y programas de formación de profesores. En este contexto, el programa que propone Rafael Bisquerra (2005) se presenta como el más completo ya que considera la EE en la formación universitaria de las y los docentes. Sin embargo, no se ha aplicado aún en España, y en Chile según los resultados de esta investigación, aún no existe una 
Estudios Pedagógicos XLVII N 1: 219-233, 2021

DOCENTES EMOCIONALMENTE INTELIGENTES. IMPORTANCIA DE LA INTELIGENCIA EMOCIONAL PARA LA

APLICACIÓN DE LA EDUCACIÓN EMOCIONAL EN LA PRÁCTICA PEDAGÓGICA DE AULA

propuesta homologa para la formación de pregrado. Cabe destacar que, aunque la temática se presenta como un aspecto importante dentro del contexto educativo, al parecer aún se considera como una especialización de postgrado y no como un requisito obligatorio en la formación inicial docente, quedando sujeta al interés particular del docente o de los establecimientos educacionales.

Actualmente se puede apreciar la existencia de variados programas que se orientan al desarrollo de habilidades socio-emocionales para el alumnado, sin embargo aunque "la educación actual es generosa en palabras referidas a la libertad interior, no se compromete con esa suerte de iluminación interna; su meta docente primordial parece ser entregar sujetos programados para ingresar a la sociedad laboral, competitiva e individualista" (Céspedes, 2018, p. 256). Esta visión sesgada del proceso educativo ha prevalecido en el tiempo, transformándose en un elemento que guía el pensamiento acerca de la calidad de la educación y que finalmente se traduce en la habilidad para responder a pruebas generalmente estandarizadas que no integran el contexto emocional de las y los estudiantes. La realidad docente es que los maestros se enfrentan a una gran diversidad de roles a desempeñar, donde sin duda el aspecto emocional y la educación integral de la personalidad de sus estudiantes es uno de los puntos más complejos y al mismo tiempo el menos atendido.

En este contexto, es imprescindible que las y los profesores lleven a cabo un "trabajo de formación personal que les ayude a tener en consideración todos los factores que influyen en el comportamiento y en la forma de aprender de sus estudiantes" (Fernández, Palomero y Teruel, 2009, p. 43), para de esta manera poder apoyarlos y acompañarlos en su crecimiento y logro de sus propias metas. Es deber de la escuela, y por ende del profesorado, crear un clima de aula favorable que estimule a los educandos a progresar, reforzando su autoestima y crecimiento integral, porque ya es sabido que el aprendizaje se da con mayor significancia cuando las y los estudiantes se sienten respetados y tratados con afecto. Desde esta perspectiva, se plantea el aprendizaje a través de las emociones como una de las finalidades de la educación, sino la más importante, ya que el clima emocional en el aula es el principal factor que explica las variaciones en el rendimiento académico de los estudiantes (Casassus, 2007).

\section{REFERENCIAS BIBLIOGRÁFICAS}

Bisquerra, R. (Coord.), Punset, E., Mora, F., García Navarro, E., López-Cassá, É., Pérez-González, J., Lantieri, L., Nambiar, M., Aguilera, P., Segovia, N. y Planells, O. (2012). ¿Cómo educar las emociones? La Inteligencia Emocional en la infancia y la adolescencia. Cuadernos Faros 6. Observatorio de salud de la infancia y la adolescencia. Hospital Sant Joan de Déu. Esplugues de Llobregat, Barcelona. Recuperado desde https://faros.hsjdbcn.org/adjuntos/2232.1-Faros\%20 6\%20Cast.pdf

Bisquerra, R. (2010). Psicopedagogía de las emociones. Madrid, España: Editorial Síntesis.

(2005). La Educación Emocional en la formación del profesorado. Revista Electrónica Interuniversitaria de Formación del Profesorado, 19(3), 95-114. Recuperado desde https:// www.redalyc.org/pdf/274/27411927006.pdf

Cabello, R., Ruiz-Aranda, D. y Fernández-Berrocal, P. (2010). Docentes emocionalmente inteligentes. Revista Electrónica Interuniversitaria de Formación del Profesorado, 13(1), 41-49. Recuperado desde https://dialnet.unirioja.es/descarga/articulo/3163455.pdf 
Campos, A. (2010). Neuroeducación: Uniendo las neurociencias y la educación en la búsqueda del desarrollo humano. Revista Digital La Educ@ción, 143. Recuperado desde http://kdoce.cl/wpcontent/uploads/2017/10/DOC1-neuroeducacion.pdf

Casassus, J. (2007). La educación del ser emocional. Santiago, Chile: Editorial Cuarto Propio.

Cejudo, J. y López-Delgado, M. (2017). Importancia de la Inteligencia Emocional en la práctica docente: un estudio con maestros. Revista Psicología Educativa. Colegio Oficial de Psicólogos de Madrid, 23(1), 29-36. Recuperado desde https://www.sciencedirect.com/science/article/pii/ S1135755X16300331

Céspedes, A. (2008). Educar las emociones. Educar para la vida. Santiago, Chile: Ediciones B. (2018). El estrés en niños y adolescentes: En busca del paraíso perdido. Santiago, Chile: Penguin Random House Grupo Editorial S. A.

Fernández, M., Palomero, J. y Teruel, M. (2009). El desarrollo socioafectivo en la formación inicial de los maestros. Revista Electrónica Interuniversitaria de Formación del Profesorado, 12(1), 33-50. Recuperado desde http://w.aufop.com/aufop/uploaded_files/articulos/1240872524.pdf

Fundación Liderazgo Chile (2018). Análisis, fundamento y programa inicial del proyecto de Ley de Educación Emocional en Chile. Recuperado desde https://docs.wixstatic.com/ugd/05c96a_950 88eff915c4b1fb6b5effa0761f15b.pdf

García, J. (2012). La Educación Emocional, su importancia en el proceso de aprendizaje. Revista Educación. Universidad de Costa Rica, 36(1), 1-24. Recuperado desde https://www.redalyc.org/ pdf/440/44023984007.pdf

Goleman, D. (1996). La Inteligencia Emocional. Barcelona: Editorial Kairos.

Hernández-Sampieri, R., Fernández, C. y Baptista, P. (2014). Metodología de la investigación. Santa Fe, México: McGraw-Hill e Interamericana Editores.

Malaisi, L. (2016). Cómo ayudar a los niños de hoy. Educación Emocional. San Juan, Argentina: Editorial Educación Emocional Argentina.

Maturana, H. (2001). Emociones y lenguaje en educación y política. Editorial Dolmen Ensayo.

Naranjo, C. (2014). Cambiar la educación para cambiar el mundo. Santiago, Chile: Editorial Cuarto Propio.

Nías, J. (1996). Thinking about feeling: the emotions in teaching. Cambridge Journal of education, 26, 293-306. Recuperado desde http://dx.doi.org/10.1080/0305764960260301

Palomero, P. (2009). Desarrollo de la competencia social y emocional del profesorado: aproximación desde la psicología humanista. Revista Electrónica Interuniversitaria de Formación del Profesorado, 12(2), 145-153. Recuperado desde https://dialnet.unirioja.es/descarga/ articulo/3039102.pdf 
